\title{
The Correlation between Flexi-bar Exercise and Trunk Muscles Strength and Body Composition in Juvenile Soccer Players
}

\begin{abstract}
The purpose of this study is to verify a correlation between flexi-bar exercise and improvement of trunk strength and body composition in juvenile soccer players. The subjects were 11 teenager juvenile soccer players who conducted flexi-bar exercise half an hour daily three times a week for eight weeks. They were tested for trunk muscles strength, body composition and correlation. The result showed that trunk muscles strength improved significantly(p<.01) and weight, Rt. arm muscle mass, Rt. leg muscle mass, and Lt. leg muscle mass(p〈.05), Lt. arm muscle mass, trunk muscle mass, and body muscle mass( $p<.01)$ improved significantly as well while Lt. arm fat mass, Rt. leg fat mass, and Lt. leg fat mass decreased significantly(p<.05). In the correlation analysis, the more trunk muscle mass and trunk muscles strength increased, the more muscle mass of body regions increased, which was a positive correlation whereas fat mass of body regions decreased, which was a negative correlation. It was verified that there was a correlation between flexi-bar exercise and improvement of trunk muscles strength and body composition for juvenile soccer players.
\end{abstract}

Key words: Juvenile soccer players; Flexi-bar; Trunk muscles strength; Body composition
Joong San Wang ${ }^{\mathrm{a}}$, Si Eun Park Hee Joon Shin', Wan Suk Choid, Hong Rae Kim , Bo Kyoung Kim ${ }^{d}$, Joo Hyun Park', Joon Hee Lee', Kyung Ok Min

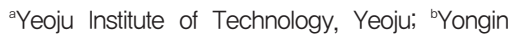
University, Yongin; 'Kyungwoon University, Gumi; "International University of Korea, Jinju; 'Suwon Women's College, Suwon; 'Cheongju University, Cheongju, Korea

Received : 21 November 2014

Revised : 15 December 2014

Accepted : 18 January 2015

Address for correspondence

Kyung Ok Min, PT, Ph.D

Department of Physical Therapy, Yongin University, 470 Samga-dong, Cheoingu, Yongin, Korea

Tel: 82-31-8020-2771

E-mail: komin@yongin.ac.kr

\section{INTRODUCTION}

While soccer is a generalized and popular sport, soccer players are experiencing injuries due to intense games, man to man defence, tactical training, and defective facilities and equipment (1, 2). In a study that performed a literature review on the injuries of Korean soccer players, Kim reported that injuries in the trunk(40.3\%) were the second most common type next to injuries in the $\operatorname{legs}(47.18 \%)(3)$.

The kicking motion in soccer requires trunk rotation(4), and therefore, causes a number of injuries due to vertebral rotation(1). Trunk muscles are an important part that is activated prior to body movements $(5,6,7)$ and trunk muscle strength is also linked with strength in the thigh region(8). Therefore, soccer players who use frequent leg movements for motions such as kicking and running should perform appropriate trunk muscle strengthening exercises to prevent injuries and maintain their physical health.

Sit-up and trunk extension exercises are generally known as the exercise method to strengthen trunk muscles. However, for curl-ups, performing them on an unstable bearing surface further increases muscle contraction in the racturs abdominis and the external oblique(9). Therefore, curlups on an unstable bearing surface can be a more effective method to strengthen trunk muscles. However, Lehman discovered that squats on a stable bearing space exhibited an overall higher level of muscle contraction in the low lumbar extensor muscle, and thus, mentioned that individual differences and varied exercise methods can produce different results(10). Therefore, static and dynamic exercises may be more effective in strengthening trunk muscles than combined strengthening exercises

At present, exercise methods to strengthen trunk muscles and improve core stability are known to be highly effective in improving strength, fat mass, 
and muscle mass. However, few studies have simultaneously implemented static and dynamic exercises. In addition, basic data on the trunk muscle strength and body composition of juvenile soccer players do not exist.

This study used the flexi-bar as the instrument to strengthen trunk muscles. The flexi-bar has the advantage of creating the co-contraction of agonist and antagonist muscles during exercises using it(11). Moreover, oscillations generated by shaking the bar stimulate trunk muscles that affect trunk stability, such as the external oblique, internal oblique, and erector spine. fexi-bar exercises are also effective for exercises of the whole $\operatorname{body}(12,13,14)$. Therefore, the purpose of this study was to develop a more effective exercise program for the management of soccer players by analyzing the effects of flexi-bar exercises on changes in trunk muscle strength and body composition and their correlations while targeting juvenile soccer players.

\section{METHODS}

\section{Subjects}

The subjects of this study were 11 students playing in a youth soccer academy located in Gyeonggi-do. This study was conducted for an eight-week period from June 30 to August 22, 2014 to identify the effects of flexi-bar exercises on changes in trunk muscle strength and body composition. Those who had problems with their skeletal and nerve systems were excluded in the selection process. Prior to the study, a school newsletter including the study's purpose and method was sent to the subjects' parents, and the study was conducted on the students who fully

Table 1. General characteristics of subjects

\begin{tabular}{|c|c|}
\hline Variable & Exercise group(M $\pm S D)$ \\
\hline $\mathrm{N}$ & 11 \\
\hline Gender & Male \\
\hline Age(year) & $10.72 \pm 90$ \\
\hline Weight(kg) & $36.63 \pm 4.48$ \\
\hline Height(cm) & $145.30 \pm 5.37$ \\
\hline Career(month) & $25.27 \pm 19.80$ \\
\hline Dominent arm & Right 10, Left 1 \\
\hline
\end{tabular}

understood the study's purpose and agreed to participate. General characteristics of the subjects are shown in Table 1.

\section{Measurement}

\section{Trunk muscles strength}

The trunk muscle strength test on the subjects was performed using the Power Track II commender(JTECH medical, USA). The strength of the rectus abdominis(RA), trunk rotator(TR), and trunk extensor(TE) was measured using Kendall's manual muscle testing(MMT)(15). During the MMT, each measurement was repeated twice and a two-minute break was provided between the measurements. In addition, a five-minute break was provided between the tests(16). All data were produced based on the average of two repeated measurement values.

\section{Body composition}

This study measured the subjects' weight, body composition, muscle mass, and fat mass using Inbody570(Inbody, Korea). Prior to the measurements, the subjects were instructed to remove any object containing metal that they were wearing and be dressed casually. Each subject stepped on a footrest located in the measuring equipment and held electrode handles with both hands. After the subject was instructed to look straight ahead while maintaining the position, the measurement as taken according to the machine's measurement order.

\section{Exercise program}

The flexi-bar(Togu / Germany) used in this study was an exercise instrument for the whole body, which could create 270 vibrations per minute $(4.6 \mathrm{~Hz})(13)$. The standard Flex-bar was used for the exercises of juvenile soccer players. For flexibar exercises, this study composed an exercise program consisting of ten exercises suitable for the study among the exercise methods suggested by Flexi-Sports(14). One set of exercises included performing each of the ten different motions for 30 seconds, and then performing the next motion without a break, and the subjects had to perform three sets of exercises with a 90 second break between the sets(Table 2). 
Table 2. Flexi-bar exercise program

\begin{tabular}{clc}
\hline $\begin{array}{c}\text { Warm-up } \\
(5 \mathrm{~min})\end{array}$ & $\begin{array}{c}\text { Flexi-bar program exercise } \\
\text { 20sec, rest } 90 \mathrm{sec}(3 \mathrm{set})\end{array}$ & $\begin{array}{c}\text { Cool-down } \\
(5 \mathrm{~min})\end{array}$ \\
\hline & 1. Butterfly Front & \\
& 2. Spine Alignment & \\
3. Plank Front(both) & Static \\
Static & 4. Bridge & \\
stretching & 5. Deep Squat & \\
& 6. 2Leg Stance(both) & \\
& 7. Hunter & \\
8. Pelvic Floor Bent Over &
\end{tabular}

\section{Data analysis}

This study produced and compared means and standard deviations for each variable by processing all collected data using the statistical software SPSS 19.0(PC). Paired t-tests were performed to compare changes in trunk muscle strength and body composition before and after the exercises. In addition, Pearson's correlation coefficient was obtained to identify correlations between trunk muscles strength and body composition. The statistical significance level was set at $\alpha=.05$.

\section{RESULTS}

This study had 11 juvenile soccer players perform flexi-bar exercises for eight weeks, three times each week and for 30 minutes each time, and then analyzed changes in their muscle strength and body composition and their correlations. The study's results are as follows.

\section{Changes of trunk muscles strength}

The trunk muscle strength of the juvenile soccer players showed statistically significant increases in the RA, TE, Rt. TR, and Lt. TR after flexi-bar exercises $(p<0.1)($ Table 3).

\section{Changes of body composition}

The body composition of the juvenile soccer players exhibited statistically significant increases in weight, Rt. arm muscle mass, Rt. leg muscle mass, Lt. leg muscle mass(p<.05), Lt. arm muscle mass, trunk muscle mass, and body muscle mass( $p<.01)$ after flexi-bar exercises, and showed statistically significant decreases in Lt. arm fat mass, Rt. leg fat mass, and Lt. leg fat mass $(p<.05)$. In particular, the statistically significant decline in the Lt. arm fat mass $(-.036 \pm .05 \mathrm{~N})$ resulted in an improvement in fat mass differences between the left and right arms(Table 4).

\section{Correlation of muscles strength and body compo- sition}

This study also examined correlations between trunk muscles strength and body composition. An increase in trunk muscle strengths resulted in corresponding increases in weight and the muscle mass of body regions $(\mathrm{r}=.046 \sim .652)$, thereby showing positive correlations. On the other hand, an increase in trunk muscle strength led to corresponding decreases in the fat mass of body regions, exhibiting negative correlations $(\mathrm{r}=-.104$ $\sim-$.837). In addition, statistically significant correlations were shown between $\mathrm{Rt}$. arm muscle mass and the TE and Rt. TR(r=.652, $\mathrm{p}=.030$; $\mathrm{r}=.613, \mathrm{p}=.045)$, between Rt. arm fat mass and the Rt. TR( $r=-.679, p=.021)$, and between Lt. arm fat mass and trunk fat mass, and the $\mathrm{RA}(\mathrm{r}=-.837$, $\mathrm{p}=.001 ; \mathrm{r}=-.738, \mathrm{p}=.010$ )(Table 5).

\section{Correlation of trunk muscle mass and body com- position}

In terms of correlations between changes in trunk muscle mass and body composition, an increase in trunk muscle strength resulted in corresponding increases in the muscle mass of body regions, which indicates positive correlations $(\mathrm{r}=.593 \sim .958)$, and led to corresponding decreases in the fat mass of body regions, showing negative correlations $(\mathrm{r}=-.109 \sim-.531)$. In particular, statistically significant positive correlations were exhibited for body muscle mass $(\mathrm{r}=.741, \mathrm{p}=.008)$ and both arm muscle mass(Rt: $\mathrm{r}=.958, \mathrm{p}=.000$, Lt: $\mathrm{r}=.955, \mathrm{p}=.000$ ), and statistically significant negative correlations were revealed for body fat mass $(\mathrm{r}=-.457, \mathrm{p}=.158)($ Table 6$)$. 
Table 3. Changes of muscle strength

\begin{tabular}{cccccc}
\hline Variable & Pre $(M \pm S D)$ & Post $(M \pm S D)$ & Change $(M \pm S D)$ & $t$ & $p$ \\
\hline Rectus abdominis & $107.89 \pm 22.77$ & $131.27 \pm 20.89$ & $23.38 \pm 17.21$ & 4.504 & $.001^{* *}$ \\
Rt trunk rotator muscle & $79.50 \pm 22.48$ & $102.12 \pm 17.27$ & $22.61 \pm 20.65$ & 3.631 & $.005^{* *}$ \\
Lt trunk rotator muscle & $73.20 \pm 18.75$ & $96.29 \pm 15.72$ & $23.08 \pm 18.92$ & 4.045 & $.002^{* *}$ \\
Trunk extensor muscle & $93.16 \pm 17.39$ & $122.80 \pm 16.06$ & $29.63 \pm 20.12$ & 4.884 & $.001^{* *}$ \\
\hline
\end{tabular}

${ }^{*} p<.05,{ }^{* *} p<.01$

Table 4. Changes of body composition

(Unit: $\mathrm{kg}$ )

\begin{tabular}{|c|c|c|c|c|c|}
\hline Variable & Pre $(M \pm S D)$ & Post (M $\pm S D)$ & 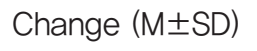 & $t$ & $p$ \\
\hline Weight & $37.03 \pm 6.72$ & $37.50 \pm 6.82$ & $.47 \pm .57$ & 2.720 & $.022^{*}$ \\
\hline Body fat mass & $6.09 \pm 3.19$ & $5.88 \pm 3.29$ & $-.20 \pm .52$ & -1.332 & .212 \\
\hline Body muscle mass & $16.39 \pm 2.61$ & $16.80 \pm 2.56$ & $.40 \pm .29$ & 4.550 & $.001^{* *}$ \\
\hline Body fat percentage & $15.63 \pm 6.55$ & $14.77 \pm 6.98$ & $-.86 \pm 1.40$ & -2.034 & .069 \\
\hline Rt. arm muscle mass & $1.24 \pm .27$ & $1.28 \pm .24$ & $.04 \pm .04$ & 3.112 & $.011^{*}$ \\
\hline Lt. arm muscle mass & $1.21 \pm .28$ & $1.26 \pm .26$ & $.05 \pm .05$ & 3.186 & $.010^{* *}$ \\
\hline Trunk muscle mass & $12.75 \pm 1.97$ & $13.06 \pm 1.83$ & $.30 \pm .29$ & 3.438 & $.006^{* *}$ \\
\hline Rt. leg muscle mass & $4.57 \pm .78$ & $4.69 \pm .70$ & $.12 \pm .15$ & 2.546 & $.029^{*}$ \\
\hline Lt. leg muscle mass & $4.54 \pm .78$ & $4.65 \pm .70$ & $.11 \pm .13$ & 2.805 & $.019^{*}$ \\
\hline Rt. arm fat mass & $.40 \pm .16$ & $.40 \pm .21$ & $.00 \pm .07$ & .430 & .676 \\
\hline Lt. arm fat mass & $.44 \pm .19$ & $.40 \pm .17$ & $-.03 \pm .05$ & -2.390 & $.038^{*}$ \\
\hline Trunk fat mass & $2.06 \pm 1.71$ & $1.94 \pm 1.77$ & $-.11 \pm .24$ & -1.579 & .145 \\
\hline Rt. leg fat mass) & $1.24 \pm .50$ & $1.17 \pm .54$ & $-.07 \pm .09$ & -2.667 & $.024^{*}$ \\
\hline Lt. leg fat mass & $1.23 \pm .49$ & $1.16 \pm .54$ & $-.07 \pm .09$ & -2.667 & $.024^{*}$ \\
\hline
\end{tabular}

${ }^{*} p<.05,{ }^{* *} p<.01$

Table 5. Correlation of muscle strength and body composition

$r(p)$

\begin{tabular}{ccccc}
\hline & Trunk extensor muscle & Lt. trunk rotator muscle & Rt. trunk rotator muscle & Rectus abdominis \\
\hline Body fat mass & $-.277(.409)$ & $-.198(.559)$ & $-2.93(.381)$ & $-.427(.190)$ \\
Weight & $.310(.354)$ & $.203(550)$ & $.399(.224)$ & $.046(.894)$ \\
Body muscle mass & $.596(.053)$ & $.430(.187)$ & $.698\left(.017^{*}\right)$ & $.504(.114)$ \\
Body fat percentage & $-.370(.263)$ & $-.257(.455)$ & $-.436(.180)$ & $-.392(.233)$ \\
Rt. arm muscle mass & $.652\left(.030^{*}\right)$ & $.370(.263)$ & $.613\left(.045^{*}\right)$ & $.469(.146)$ \\
Lt. arm muscle mass & $.416(.203)$ & $.102(.766)$ & $.405(.217)$ & $.502(.116)$ \\
Trunk muscle mass & $.505(.113)$ & $.262(.437)$ & $.455(.160)$ & $.446(.169)$ \\
Rt. leg muscle mass & $.400(.222)$ & $.274(0.414)$ & $416(.203)$ & $.376(.254)$ \\
Lt. leg muscle mass & $.438(.178)$ & $.287(.391)$ & $.444(.172)$ & $.373(.259)$ \\
Rt. arm fat mass & $-.237(.483)$ & $.104(.760)$ & $-.679\left(.021^{*}\right)$ & $-.506(.112)$ \\
Lt. arm fat mass & $-.311(.351)$ & $.073(.832)$ & $-.118(730)$ & $-.837\left(.001^{* *}\right)$ \\
Trunk fat mass & $-.518(.102)$ & $-.187(.582)$ & $-.552(.079)$ & $-.738\left(.010^{* *}\right)$ \\
Rt. leg fat mass & $-.145(.670)$ & $.097(.777)$ & $-.477(.138)$ & $-.377(.252)$ \\
Lt. leg fat mass & $-.417(.202)$ & $-.104(.761)$ & $-.541(.085)$ & $-.408(.212)$ \\
\hline
\end{tabular}

${ }^{*}$ p $<.05,{ }^{* *} p<.01$ 
Table 6. Correlation of trunk muscle mass and body

\begin{tabular}{cc} 
composition & $r(p)$ \\
\hline Variable & Trunk muscle mass \\
\hline Body fat mass & $-.457(.158)$ \\
Weight & $.229(.499)$ \\
Body muscle mass & $.741\left(.008^{* *}\right)$ \\
Body fat percentage & $-.625\left(.040^{*}\right)$ \\
Rt. arm muscle mass & $.958\left(.000^{* *}\right)$ \\
Lt. arm muscle mass & $.955\left(.000^{* *}\right)$ \\
Rt. leg muscle mass & $.639\left(.034^{*}\right)$ \\
Lt. leg muscle mass & $.593(.054)$ \\
Rt. arm fat mass & $-.531(.093)$ \\
Lt. arm fat mass & $-.109(.750)$ \\
Trunk fat mass & $-.349(.293)$ \\
Rt. leg fat mass & $-.233(.491)$ \\
Lt. leg fat mass & $-.344(.301)$
\end{tabular}

${ }^{*} p<.05,{ }^{* *} p<.01$

\section{DISCUSSION}

By the nature of soccer as a sport, soccer players who frequently kick a ball using trunk rotation $(1,4)$ experience many injuries due to vertebral rotation(1) and exhibit coupled movements of the lumbar spine, which differ from general vertebral movements(17). Therefore, soccer players should have their trunk muscles strengthened and developed in a coordinated manner through appropriate exercises and management from their early ages.

Meanwhile, as juvenile soccer players also show the correlation between injuries and asymmetric trunk stability(18), soccer players should perform exercises that can increase the stability of trunk muscles from their early ages. Among muscles that work for trunk stabilization, the co-contraction of the external oblique, internal oblique, and transverse abdominis increases muscle activation and abdominal pressure, thereby increasing the overall stiffness of trunk muscles and improving trunk stability(19). Therefore, the flexi-bar exercises applied in this study are likely to be effective for the balanced development of trunk muscles and the prevention of sports injuries.

The flexi-bar used in this study is widely used in fitness centers and rehabilitation therapies to improve coordination capability and muscle strength(12). However, previous studies that used flexi-bar exercises are hardly available. Among the relevant previous studies reported thus far, a study by Chung compared the muscle activation of trunk muscles during flexi-bar and non-flexi-bar exercises(13). During flexi-bar exercises, the muscle activation of the internal oblique showed a statistically significant difference in the quadruped position and the muscle activation of the internal oblique and erector spine showed statistically significant differences in the bridge position. In addition, the muscle activation of the external oblique and internal oblique exhibited statistically significant differences in the standing position. Based on these results, the author suggested that flexi-bar exercises are effective in strengthening trunk muscles.

In this study, the strength of trunk muscles statistically significantly increased in the $R A(p=.001)$, Lt. $\mathrm{TR}(\mathrm{p}=.005)$, Rt. $\mathrm{TR}(\mathrm{p}=.002)$, and $\mathrm{TE}(\mathrm{p}=.001)$ after the given exercises, which confirmed that flexi-bar exercises can be a highly effective method to strengthen trunk muscles.

In terms of changes in the body composition of juvenile soccer players, the muscle mass of body regions increased and the fat mass of body regions decreased. In specific, the variables of weight, trunk muscle mass, Rt. arm muscle mass, Rt. leg muscle mass, Lt. leg muscle mass(p<.05), Lt. arm muscle mass, and body muscle mass showed statistically significant increases ( $p<.01)$, whereas Lt. arm fat mass, Rt. leg fat mass, and Lt. leg fat mass exhibited statistically significant declines (p<.05). In particular, the statistically significant decline in Lt. arm fat mass resulted in an improvement in fat mass differences between the right and left arms. Athletes with high levels of fat mass are associated with reduction in lumbar flexion and extension and decreases in the height of vertebral discs(20). Therefore, increases in trunk muscle mass and decreases in trunk fast mass derived from using flexi-bar exercises can have positive effects on maintaining normal levels of vertebral flexibility for juvenile soccer players.

In addition, this study examined correlations between changes in trunk muscle mass and body composition after flexi-bar exercises. As a result, an increase in trunk muscle mass led to corresponding increases in the muscle mass of body regions, which shows positive correlations, and resulted in corresponding decreases in the fat mass of body regions, which shows negative correlations. In particular, statistically significant positive correlations were exhibited for body muscle mass and both arm muscle mass, and a statistically significant negative correlation was revealed 
for body fat percentage. These results indicated that changes in the trunk muscle mass of juvenile soccer players after performing flexi-bar exercises have overall positive impacts on increasing muscle mass and decreasing fat mass.

Regarding the relationship between changes in trunk muscle strength and body composition, an increase in trunk muscles strength led to corresponding increases in weight and the muscle mass of body regions, thereby showing positive correlations, and resulted in decreases in the fat mass of body regions, thereby exhibiting negative correlations.

Trunk muscles are characterized by being activated prior to leg movements(21). Improvements in trunk muscle strength increase extension strength and flexion strength of the thigh, and help strengthen calf muscles(8). Therefore, the strengthening of trunk muscles will also become a highly important factor in improving athletic performance.

This study was conducted on only juvenile soccer players, and thus has the limitation that its results cannot be generalized for soccer players in other age groups and a control group was not established. However, given that previous studies that examined the trunk muscle strength and body composition of Korean soccer players do not exist, the results of this study may still become basic data for future relevant studies.

\section{CONCLUSION}

This study analyzed the effects of an eight-week flexi-bar exercise program on the trunk muscle strength and body composition of 11 soccer players and correlations between the variables, and derived the following results. The flexi-bar exercises statistically significantly improved the strength of trunk muscles and the muscle mass of arms and legs, and statistically significantly decreased the fat mass of body regions. In terms of correlations between the variables, increases in trunk muscle mass and trunk muscle strength resulted in corresponding increases in the muscle mass of body regions, thereby exhibiting positive correlations. On the other hand, negative correlations were revealed for body fat mass.

\section{REFERENCES}

1. Yang SH. Study of Exercise Injuries among the Middle, High Soccer Players. Mokpo University 2006.

2. Ha BS. Comparative analysis of the injury types of soccer players. KyongGi University 2002.

3. Kim SH. A study of reviewing a trend of korean sports injury literatures. Journal of Sport and Leisure Studies 2008; 34: 1029-1039.

4. Kim SB. A biomechanical analysis of the leg segments during a soccer instep kick motion. Dankook University 1996.

5. Akuthota V, Ferreiro A, Moore T, Fredericson M. Core stability exercise principles. Curr Sports Med Rep 2008; 7(1): 39-44.

6. Brill PW, Couzens GS. The core program. Bantam 2003.

7. Neumann, D. Kinesiology of the musculoskeletal system: Foundations for rehabilitation. st louis, MO: Mosby 2010.

8. Hong SM. The relationships among isokinetic trunk muscle, knee, and ankle strengths of soccer players. Yeungnam University 2002.

9. Vera-Garcia FJ, Grenier SG, McGill SM. Abdominal muscle response during curl-up on both stable and labile surfaces. Phys Ther 2000; 80(6): $564-569$.

10. Lehman GJ. An unstable support surface isnot a sufficient condition for increases in muscle activity during rehabilitation exercise. $\mathrm{J}$ Can Chiropr Assoc 2007; 5(13): 139-143.

11. Kisner C, Colby LA: Therapeutic Exercise: foundations and techniques(5th ed). F.A Philadelphia: Davis Company 2007.

12. Mileva KN, Kadr M, Amin N, Bowtell JL. Acute effects of Flexi-bar vs Sham-bar exercise on muscle electromyography activity and performance. J Strength Cond Res 2010; 24: 737 - 748.

13. Chung JS. Effect of flexi-bar exercise on trunk muscles activity according to posture change in healthy adult. Catholic University of Daegu 2013.

14. www.flexi-sports.com

15. Kendall FP, Provance PG, McCreary EK. Muscles: Testing and function with posture and pain. 4th ed. Baltimore, Williams \& Wilkins 2005.

16. Dunn JC, Maura D. Interrater reliability of knee muscle forces obtained by hand-held dynamometer from elderly subjects with degenerative back pain. J Geriatr Phys Ther 2003; 26(3): 23-29. 
17. Moon OK. A study of spinal activity pattern in football, baseball players and general students. Yongin University 2011.

18. Nakazawa R, Endo Y, Sakamoto M. (2013). The relationship between trunk function and injury among junior high school soccer players. J Phys Ther Sci 2013; 25(7): 775-777.

19. McGill SM. Low back stability: from formal description to issues for performance and rehabilitation. Exerc Sport Sci Rev 2001; 29(1): 26-31.
20. Räty HP, Battié MC, Videman T, Sarna S. Lumbar mobility in former élite male weightlifters, soccer players, long-distance runners and shooters. Clin Biomech(Bristol, Avon) 1997; 12(5): 325-330.

21. Hodges Pw, Richardson CA. Delayed postural contraction of transversus abdominis in low back pain associated with movement of the lower limb. J Spinal Disord 1998; 11(1): 46-56. 